\title{
Impacto do Programa Pré-Esportivo da Equoterapia na Flexibilidade, Força e Equilíbrio em Indivíduos com Deficiência Intelectual
}

\author{
Impact of the Equoterapy Sporting Program on Muscle Flexibility, Strength and Balance in \\ Individuals With Intellectual Disabilities
}

Impacto del Programa de Equinoterapia Predeportiva en la Flexibilidad, la Fuerza y el Equilibrio en Personas con Discapacidades Intelectuales

Valeria Silva Monteiro ORCID: https://orcid.org/0000-0003-4208-0654 Universidade Federal do Triângulo Mineiro, Brasil E-mail: valeriasmonteiro69@gmail.com

Lara Aparecida de Paula Maia

ORCID: https://orcid.org/0000-0001-7299-8904 Universidade Federal do Triângulo Mineiro, Brasil E-mail: laraapmaia@outlook.com

Delivânia da Rocha Meireles Oliveira

ORCID: https://orcid.org/0000-0002-3970-1037 Universidade Federal do Triângulo Mineiro, Brasil E-mail: mdelivania@gmail.com

Janaine Brandão Lage

ORCID: https://orcid.org/ 0000-0002-1712-5593

Universidade Federal do Triângulo Mineiro, Brasil E-mail: ja.bl@terra.com.br

Leonardo Augusto Lombardi ORCID: https://orcid.org/0000-0002-9735-8804 Universidade Federal do Triângulo Mineiro, Brasil E-mail: leonardo.lombardi@uftm.edu.br Rodrigo César Rosa

ORCID: https://orcid.org/0000-0003-3157-0826 Universidade Federal do Triângulo Mineiro, Brasil E-mail: rodrigo.rosa@uftm.edu.br

Fabrizio Antônio Gomide Cardoso

ORCID: https://orcid.org/0000-0001-7520-2879 Universidade Federal do Triângulo Mineiro, Brasil E-mail: fabrizio.cardoso@uftm.edu.br

Ana Paula Espindula

ORCID: https://orcid.org/0000-0002-9282-4482 Universidade Federal do Triângulo Mineiro, Brasil E-mail: ana.espindula@uftm.edu.br

\section{Resumo}

O objetivo foi verificar o impacto do programa pré-esportivo da equoterapia na flexibilidade, força e equilíbrio de pessoas com deficiência intelectual. Participaram do estudo seis indivíduos com idade média de 17,5 $\pm 2,07$ anos. Os atendimentos foram realizados uma vez por semana, por 30 minutos, a sela inglesa foi utilizada com o cavalo ao trote. O bloco de Wells foi usado para a avaliação da flexibilidade, o teste de Sentar e Levantar e o dinamômetro lombar para a avaliação de força muscular, e o teste Clínico de Integração Sensorial do Equilíbrio. As diferenças com valor p igual ou inferior a 5\% foram consideradas estatisticamente significativas. Os resultados apresentaram significância na flexibilidade muscular $(\mathrm{p}=0,026)$, força muscular por meio do teste de Sentar e Levantar $(\mathrm{p}=0,004)$, dinamômetro lombar $(\mathrm{p}=0,014)$ e equilíbrio $(\mathrm{p}=0,045)$, após 10 atendimentos. Concluímos que o programa pré esportivo da equoterapia contribuiu para o desenvolvimento e desempenho físico desses sujeitos.

Palavras-chave: Amplitude de movimento articular; Deficiência intelectual; Equilíbrio postural; Esporte; Força muscular; Terapia Assistida por cavalos.

\section{Abstract}

The objective was to verify the impact of the equine therapy sports program on the flexibility, strength and balance of people with intellectual disabilities. Six individuals participated in the study with a mean age of $17.5 \pm 2.07$ years. The attendance were made once a week, for 30 minutes, the english saddle was used with the horse at a trot. Wells' block 
was used to assess flexibility, the Sit and Stand test and the lumbar dynamometer to assess muscle strength, and the Clinical Balance Sensory Integration test. Differences with a p-value equal to or less than $5 \%$ were considered statistically significant. The results showed significance in muscle flexibility $(p=0.026)$, muscle strength through the Sit and Stand test $(p=0.004)$, lumbar dynamometer $(p=0.014)$ and balance $(p=0.045)$, after ten attendance. We conclude the hippotherapy program for sports contributed to the development and physical performance of these subjects.

Keywords: Range of motion articulate; Intellectual disability; Postural Balance; Sport; Muscle Strength; Equineassisted therapy.

\section{Resumen}

El objetivo fue verificar el impacto del programa pre-deportivo de hipoterapia sobre la flexibilidad, la fuerza y el equilibrio de las personas con discapacidad intelectual. Seis individuos participaron en el estudio con una edad media de 17,5 \pm 2,07 años. Las consultas se realizaron una vez a la semana, durante 30 minutos, se utilizó la silla inglesa con el caballo al trote. El bloque de Wells se utilizó para evaluar la flexibilidad, la prueba Sit and Stand y el dinamómetro lumbar para evaluar la fuerza muscular y la prueba de Integración Sensorial del Balance Clínico. Las diferencias con un valor de $\mathrm{p}$ inferior al $5 \%$ se consideraron estadísticamente significativas. Los resultados mostraron significancia en flexibilidad muscular $(\mathrm{p}=0.026)$, fuerza muscular a través del test Sit and Stand $(\mathrm{p}=0.004)$, dinamómetro lumbar $(\mathrm{p}=$ $0.014)$ y equilibrio $(\mathrm{p}=0.045)$, luego de 10 visitas. Concluimos que el programa de hipoterapia pre-deportiva contribuyó al desarrollo y rendimiento físico de estos sujetos.

Palabras clave: Rango de movimiento articular; Discapacidad intelectual; Equilibrio postural; Deporte; Fuerza muscular; Terapia asistida por caballos.

\section{Introdução}

A deficiência intelectual (DI) é um transtorno no desenvolvimento detectado, até os 18 anos de idade, sendo, portanto, evidenciada por funcionamento limitado da capacidade cognitiva. Aproximadamente 1,4\% da população brasileira é acometida por esse transtorno (Shäfer et al., 2019).

Essa deficiência pode ser categorizada de acordo com as habilidades conceituais, sociais e práticas que permitem adaptação do indivíduo ao ambiente, podendo ser considerada leve, moderada, grave ou profunda. A capacidade de compreensão da pessoa com DI de grau moderado é identificada por distúrbios no domínio da atenção, da capacidade de raciocínio lento, das falhas perceptivas e da dificuldade em associar. Esses indivíduos podem possuir dificuldade na alocução e no entendimento de tratos sociais, periodicamente mudam de conduta e possuem necessidade de seguir uma rotina (Tomaz et al., 2017). Para Schwartzman e Lederman (2017), indivíduos com DI apresentam desordens na comunicação, no relacionamento pessoal e social e nas funções nervosas e musculares.

Entre as possibilidades de métodos terapêuticos encontra-se a equoterapia, a qual utiliza como recurso de intervenção o cavalo, que aborda de maneira integral âmbitos da saúde, educação e equitação, afim de aprimorar aspectos biológicos e domínios psicológicos e sociais de pessoas com deficiência. A equoterapia envolve quatro programas básicos dentro de sua abordagem, sendo eles: hipoterapia, educação e reeducação, pré-esportivo e prática esportiva paraequestre (Ande, 2019; Araújo et al., 2021).

O cavalo dispõe de três andaduras diferentes: o passo, o trote e o galope, e estas são empregadas com finalidades e propriedades particulares da modalidade em que os praticantes estão inseridos dentro da equoterapia (Silva et al., 2019). Estas propriedades específicas se dão pelo fato dos movimentos desempenhados pelo cavalo estimularem o indivíduo a executar ajustes tônicos no tecido muscular esquelético (Espindula et al., 2012). O programa pré-esportivo utiliza como andadura principal o trote, movimento saltado a dois tempos, exigindo do praticante uma maior agilidade (Wickert, 1999). Portanto, o praticante que está inserido neste programa deve ser capaz de apresentar independência para a condução, exercendo maior influência sobre o cavalo, deste modo desenvolve capacidade para realizar modalidades específicas do hipismo (Ande, 2019).

Esta pesquisa se fez necessária, após realizar um levantamento dos estudos e observar que não foram encontradas pesquisas que avaliam a flexibilidade muscular, a força muscular e o equilíbrio postural no programa pré-esportivo da equoterapia em praticantes com DI. Por conseguinte, pesquisas que se empenham em fornecer conhecimentos detalhados de 
atividades esportivas alternativas para a crescente população de pessoas com deficiência física e/ou intelectual são importantes, sob o ponto de vista científico e social. Assim a hipótese desse estudo é que indivíduos com DI inseridos no programa préesportivo da equoterapia melhoram a flexibilidade muscular, a força muscular e o equilíbrio postural. Dessa forma, o objetivo desta pesquisa foi avaliar o impacto do programa pré-esportivo da equoterapia na flexibilidade muscular, força muscular e equilíbrio postural em indivíduos com DI após 10 atendimentos.

\section{Metodologia}

Estudo analítico, quantitativo e prospectivo (Pereira et al., 2018), aprovado pelo Comitê de Ética e Pesquisa (CEP) sob protocolo n 3.290.656 e Comissão de Ética no Uso de Animais (CEUA) 426/2017. Os pais e/ou responsáveis pelos indivíduos que participaram da pesquisa receberam esclarecimentos quanto aos objetivos, procedimentos realizados pelo estudo e aqueles que consentiram, assinaram o Termo de Consentimento Livre e Esclarecido (TCLE).

As coletas de dados foram realizadas no Centro de Equoterapia Dr Guerra da Associação de Pais e Amigos dos Excepcionais (APAE). A amostra foi constituída por seis indivíduos saudáveis com DI moderada, sendo dois participantes do gênero masculino e quatro do gênero feminino, com média de idade de 17,5 \pm 2,07 anos, conforme caracterização da amostra apresentada na Tabela 1, com condições prévias para avançar no programa pré-esportivo, considerando que os indivíduos foram oriundos dos programas de hipoterapia e educação/reeducação, os quais utilizavam o cavalo na andadura ao passo e a sela australiana como material de montaria. Todos os participantes foram selecionados por conveniência, ressaltando suas potencialidades evolutivas e não apresentavam doenças associadas.

Tabela 1. Caracterização da amostra.

\begin{tabular}{cccccc}
\hline Indivíduos & $\begin{array}{c}\text { Gênero } \\
\text { F/M }\end{array}$ & $\begin{array}{c}\text { Idade } \\
(\mathbf{a n o s})\end{array}$ & $\begin{array}{c}\text { Altura } \\
(\mathbf{c m})\end{array}$ & $\begin{array}{c}\text { Peso } \\
(\mathbf{K g})\end{array}$ & $\begin{array}{c}\text { IMC } \\
\left(\mathbf{K g} / \mathbf{m}^{2}\right)\end{array}$ \\
\hline 1 & $\mathrm{~F}$ & 18 & 1,56 & 82 & 21,3 \\
2 & $\mathrm{~F}$ & 18 & 1,78 & 86 & 27,2 \\
3 & $\mathrm{M}$ & 15 & 1,69 & 58 & 20,3 \\
4 & $\mathrm{~F}$ & 20 & 1,65 & 61 & 22,4 \\
5 & $\mathrm{M}$ & 19 & 1,65 & 60 & 22 \\
6 & $\mathrm{~F}$ & 15 & 1,76 & 85,5 & 27,6 \\
& & & & & $\mathbf{2 3 , 4 6}$ \\
& Média & $\mathbf{1 7 , 5}$ & $\mathbf{1 , 6 8}$ & $\mathbf{7 2 , 0 8}$ & $\mathbf{3 , 1 3}$ \\
\hline
\end{tabular}

Média e desvio padrão $( \pm)$ das medidas antropométricas [peso $(\mathrm{Kg})$, estatura $(\mathrm{cm})$, gênero feminino (F) e masculino $(\mathrm{M})$ e índice de massa corporal $\left(\mathrm{Kg} / \mathrm{m}^{2}\right)$.

Fonte: Elaborado pelos autores.

As avaliações da flexibilidade muscular, da força muscular e equilíbrio postural foram realizadas antes do primeiro atendimento e após o décimo atendimento do programa Pré-esportivo da equoterapia com o cavalo na andadura ao trote. 
Para avaliar a flexibilidade muscular foi utilizado o Bloco de Wells com dimensões de $35 \mathrm{~cm}$ de altura e largura e 40 $\mathrm{cm}$ de comprimento com régua padrão. A avaliação foi realizada de maneira ativa pelos praticantes, os quais foram posicionados sentados de frente para o Bloco de Wells, com a planta dos pés em pleno contato com a face anterior do bloco, membros inferiores com extensão de joelhos e com os quadris fletidos. Após o posicionamento, com os membros superiores em extensão de cotovelo apoiados sobre a régua e com uma mão sobre a outra, os praticantes foram orientados a realizar flexão de tronco, executando o maior alcance de movimento sobre a régua do bloco de Wells. Os valores foram obtidos a partir da realização de três medidas, com intervalo de 10 segundos entre as aferições, para a extração da média, expressos em centímetros (Bertolla et al., 2007; Espindula et al., 2012).

$\mathrm{Na}$ avaliação da força muscular foram aplicados duas avaliações, o teste de Sentar e Levantar da cadeira e avaliação por meio do dinamômetro lombar da marca Oswaldo Filizola ${ }^{\circledR}$, modelo Crown Dorsal. O teste de Sentar e Levantar da cadeira, para mensurar a força dos membros inferiores, foi realizado por meio da apuração de quantas vezes o participante conseguiu sentar e levantar, sem apoio e auxílio dos braços durante 30 segundos (número de repetições máximas), em uma cadeira com encosto dorsal e sem apoio para os braços (Bohannon, 1995). Para avaliação da força lombar isométrica estática foi utilizado o dinamômetro lombar que possui uma célula de carga acoplada a uma plataforma de base com puxador para as mãos com capacidade máxima de 200,0 Quilograma-força (kgf). Para execução do teste, o participante foi posicionado em pé com as mãos apoiadas no puxador com semiflexão dos joelhos, evitando uma sobrecarga na região lombar. Para o teste, foi solicitado que o indivíduo realizasse o máximo de força possível com objetivo de elevar uma alavanca (Eichinger et al., 2016). Cada participante realizou três tentativas, com intervalo de 10 segundos entre elas, para estimar a média.

O Teste Clínico de Integração Sensorial do Equilíbrio (CTSIB) tem como objetivo avaliar três principais sistemas sensoriais envolvidos no equilíbrio (visão, vestibular e somatossensorial). O teste é realizado em seis condições sensórias com os olhos abertos ou fechados e em dois tipos de superfície: estável (chão) e instável (superfície de espuma) . O participante foi orientado a manter-se pelo tempo de 30 segundos em cada uma das condições, sendo realizadas três medidas, levando em consideração a média, com intervalo de 10 segundos entre as repetições. Para padronização do solo instável, foi utilizado uma espuma de densidade 28, com 7,5 cm de altura e 50 cm de comprimento e largura (Steindl et al., 2006; Riley \& Clark, 2003). O teste foi realizado na seguinte ordem: Condição 1: olhos abertos, pés juntos, em superfície estável. Condição 2: olhos fechados com venda, pés juntos, em superfície estável. Condição 3: olhos abertos, pés juntos, sobre superfície instável. Condição 4: olhos fechados com venda, pés juntos, em superfície instável. Condição 5: olhos abertos, pés em posição de Tandem (calcanhar do pé não dominante posicionado logo à frente do hálux do pé dominante) em superfície instável. Condição 6: olhos abertos, em posição unipodal (membro dominante apoiado) em superfície instável.

As avaliações foram realizadas em uma sala fechada, climatizada $\left(25^{\circ} \mathrm{C}\right)$ e sem interferências de pessoas ou ruídos que pudessem desconcentrar os avaliados. A superfície de apoio foi plana e não escorregadia, o avaliado estava descalço e sem meias, trajando roupas confortáveis que não restringiram o arco de movimento das articulações e o avaliador posicionava à frente e em diagonal ao avaliado, procurando uma visão completa de seus movimentos a fim de fornecer segurança ao individuo. As avaliações foram feitas sempre por um mesmo avaliador.

Os atendimentos da equoterapia no programa pré-esportivo foram realizados uma vez por semana, com duração de trinta minutos. Os atendimentos transcorreram em um redondel com superfície de areia com $4 \mathrm{~cm}$ de profundidade, descoberto, cercado com postes de madeira e medindo 14 metros de diâmetro. O material de montaria utilizado foi à sela inglesa, com cavalo na andadura ao trote. O cavalo selecionado para o estudo foi um animal adulto de 19 anos da raça PaintHorse com características de 1,44 cm de altura, $430 \mathrm{~kg}$ de peso. Durante todos os atendimentos os praticantes utilizavam os equipamentos de proteção individual (EPIs) como capacete, calça comprida e calçados fechados, preferivelmente com pequenos saltos, favorecendo o posicionamento seguro e correto, sendo ponta de pé apoiada e projetada pra a cima e calcanhares projetados 
para baixo, evitando assim, serem empurrados para dentro dos estribos e ficarem presos durante a realização do trote. Os atendimentos foram realizados sempre por dois profissionais.

As análises estatísticas foram realizada utilizando o software Sigma-Stat $3.5^{\circledR}$. A normalidade dos dados foi verificada por meio do teste de Shapiro Wilk e para homogeneidade das variâncias o teste de Bartlett. Para os resultados não paramétricos utilizou-se o teste de Kruskal-Wallis seguido do teste de Friedman e para os resultados paramétricos, o teste t. As diferenças com valor p igual ou inferior a $5 \%$ foram consideradas estatisticamente significativas.

\section{Resultados}

Os resultados da avaliação da flexibilidade ( $\mathrm{p}=0,026)$, da força muscular dos membros inferiores $(\mathrm{p}=0,004)$, e da força lombar $(\mathrm{p}=0,014)$ apresentaram valores estatísticos significativos, após os 10 atendimentos (Tabela 2).

Tabela 2. Avaliação da flexibilidade e força muscular

\begin{tabular}{ccccc}
\hline & & Flexibilidade & TSL & Dinamômetria \\
\hline Antes & Média $( \pm)$ & $24,167 \pm(13,091)$ & $9,833 \pm 3,764$ & $35,000 \pm 8,854$ \\
& & & & \\
\multirow{2}{*}{ Após } & Média $( \pm)$ & $28,667 \pm 10,893$ & $13,667 \pm 3,615$ & $47,500 \pm 14,167$ \\
& Valor de p & $0,026^{*}$ & $0,004^{*}$ & $0,014^{*}$ \\
\hline
\end{tabular}

Legenda: TSL (teste de Sentar e Levantar da cadeira); \pm (desvio padrão). Teste estatístico: amostras não paramétricas, Kruskal-Wallis; amostras paramétricas, teste t. Estatístico para $\mathrm{p}<0,05^{*}$.

Fonte: Elaborado pelos autores

$\mathrm{Na}$ avaliação do equilíbrio, foi possível identificar que a condição 5, olhos abertos com os pés em posição de Tandem em superfície instável, apresentou resultado significativo após os 10 atendimentos (p=0,045), conforme Tabela 3. 
Tabela 3. Avaliação do equilíbrio - CTSIB

\begin{tabular}{|c|c|c|c|}
\hline CTSIB -Condição & $\mathrm{N}^{\mathrm{o}}$ & Média \pm ou Mediana (Min-Max) & Valor de $\mathrm{p}$ \\
\hline 1 - Antes & 6 & $30(30-30)$ & \\
\hline 1 - Após & 6 & $30(30-30)$ & $=0,699$ \\
\hline 2 - Antes & 6 & $29(14-30)$ & \\
\hline 2 - Após & 6 & $30(30-30)$ & $=0,180$ \\
\hline 3 - Antes & 6 & $29(19-30)$ & \\
\hline 3 - Após & 6 & $30(30-30)$ & $=0,180$ \\
\hline 4 - Antes & 6 & $20,667 \pm 11,928$ & \\
\hline 4 - Após & 6 & $30,000 \pm 0,000$ & $=0,113$ \\
\hline 5 - Antes & 6 & $7,500 \pm 9,203$ & \\
\hline 5 - Após & 6 & $16,167 \pm 6,014$ & $=0,045^{*}$ \\
\hline 6 - Antes & 6 & $7,833 \pm 7,223$ & \\
\hline 6 - Após & 6 & $9,000 \pm 6,928$ & $=0,553$ \\
\hline
\end{tabular}

Legenda: CTSIB (Clinical Test of Sensory Integration of Balance); $\mathrm{N}^{\mathbf{o}}$ (número de participantes); SD (desvio padrão); Min (mínimo); Max (máximo). Teste estatístico: amostras não paramétricas, Kruskal-Wallis; amostras paramétricas, teste t. Estatístico para $\mathrm{p}<0,05^{*}$.

Fonte: Elaborado pelos autores

\section{Discussão}

A partir do objetivo proposto neste estudo, que foi verificar o impacto do programa pré-esportivo da equoterapia na flexibilidade muscular, na força muscular e no equilíbrio postural em indivíduos com DI, pode-se concluir que houve melhoras nos parâmetros avaliados, validando a hipótese deste estudo.

O programa pré-esportivo da equoterapia apresentou importante papel no desenvolvimento e desempenho funcional de indivíduos com DI moderada. O aumento da flexibilidade muscular em nossos resultados estão em consonância com achados da literatura, em que programas regulares de atividades físicas, como a equoterapia, evidenciam melhora dos níveis de flexibilidade (Espindula et al., 2012). As habilidades adquiridas pelo indivíduo relacionadas à flexibilidade influenciam seu potencial funcional e seu controle postural global quando montado a cavalo (Moraes et al ., 2015). O indivíduo, no processo de desenvolvimento das capacidades físicas e exploração do corpo sobre o cavalo, amplia as possibilidades direcionadas à flexibilidade para melhor desempenho de suas atividades de vida diária (Gross et al., 2019). Assim inferimos que o aumento da flexibilidade muscular deu-se mediante ao programa pré-esportivo exigir constantes extensões de membros inferiores para conduzir e direcionar o animal, assim como a temperatura do corpo do cavalo, que em condições normais para o animal adulto é de $38^{\circ} \mathrm{C}$, em contado com o praticante.

Em estudos direcionados, a evolução da montaria e cinética de cavalos em deslocamento, descreve-se o trote como um andamento simétrico em dois tempos que ocorre em diagonal, com grau moderado a intenso de impulsão, auxiliando no monitoramento da qualidade dos programas de exercícios aos praticantes (Silva, 2018). Em adição a sela inglesa mediante sua ergonomia específica (Ande, 2019), favorece a anteroversão da pelve, promovendo a realização do trote direcionados à praticas pré-esportivas e esportivas (Pereira, Silva \& Mazo, 2015). Inferindo a necessidade de ajustes tônicos e aumento da força muscular para se manter sentado sobre o cavalo. A escolha do material de montaria de acordo com o comprometimento físico e 
ou intelectual do sujeito e com o objetivo terapêutico proposto, é de fundamental importância para alcançar resultados positivos, enfatizados em estudos descritos na literatura que avaliaram o aumento da atividade elétrica muscular, por meio do eletromiográfo de superficie, a utilização de alguns materiais de montaria e a colocação ou não dos pés nos estribos para diversos processos patológicos gerais (Laje et al., 2020; Fernandes et al., 2018; Espindula et al., 2014). A força é sustentada por uma combinação de fatores morfológicos e neurais, incluindo a área e arquitetura da seção transversal muscular, rigidez musculotendinosa, recrutamento da unidade motora, codificação da taxa, sincronização da unidade motora e inibição neuromuscular. Treinamento bilateral, treinamento excêntrico e carga excêntrica acentuada e treinamento de resistência variável podem produzir as adaptações de força mais abrangentes. Exercícios de peso corporal, exercícios de isolamento, exercícios pliométricos, exercícios unilaterais e treinamento com kettlebell podem ser limitados em seu potencial para melhorar a força máxima, mas ainda são relevantes para o desenvolvimento da força ao desafiar a expressão de força limitada no tempo e desafiar diferencialmente as demandas motoras. Atletas mais fracos devem se concentrar no desenvolvimento de força antes de enfatizar o treinamento do tipo potência (Suchomel et al., 2018). Assim, concluímos que a andadura ao trote do cavalo e o material de montaria usado nesse estudo exigiu do praticante recrutamento de unidades motoras dos membros inferiores e região lombar para se ajustar a movimentação do cavalo, o que infere a obtenção do aumento de força nessas regiões.

O equilíbrio dos praticantes com DI avaliados por meio do teste CTSIB apresentou respostas favoráveis por influência do programa pré-esportivo da Equoterapia com melhora significativa para a condição cinco. Estudos aplicando o CTSIB enfatizam que são essenciais as informações aferentes dos sistemas vestibular, visual, proprioceptivo e interoceptivo, que promovem a interação do corpo com o espaço e possibilitam a manutenção do equilíbrio postural (Carneiro et al., 2010). Reforçando que o equilíbrio corporal é alcançado quando forças internas e externas atuam permitindo o controle para que o indivíduo permaneça na posição desejada (equilíbrio estático) ou se mova de maneira controlada (equilíbrio dinâmico) (Ribeiro et al., 2016). Júnior et al (2018) comparou o equilíbrio postural de crianças com DI com grupo de crianças típicas, foi possível verificar que, nos parâmetros avaliados na condição apoio bipodal com o unipodal, as crianças com DI apresentaram resultados semelhantes ou superiores ao grupo controle, onde estes resultados sugerem que a DI parece não ser um fator limitante no desempenho do equilíbrio postural estático desses indivíduos. Corroborando com nossos estudos, em que inferimos que a capacidade de adaptação do praticante ao conduzir o cavalo ao trote em superfície de areia, os diversos estímulos visuais encontrados em um centro de equoterapia, como outros cavalos próximos, atendimentos acontecendo simultaneamente e paisagens, além das constantes movimentações articulares globais, em especial a da região de pescoço nos sentidos láterolateral, crâniocaudal e rotacional, tenha permitido o estímulo dos sistemas sensoriais (visão, vestibular e somatossensorial), levando a melhora do equilíbrio.

As pesquisas publicadas direcionadas ao programa pré-esportivo ainda são escassas, embora a equoterapia tenha se destacado no âmbito científico, em especial à hipoterapia. Contudo, diante dos resultados encontrados, concluímos que o pré esportivo da equoterapia influenciou no aumento da flexibilidade e força muscular, assim como a melhora do equilíbrio postural de indivíduos com DI.

\subsection{Limitações}

Como limitações do presente estudo descrevemos o número baixo de sujeitos incluídos na pesquisa, e a coleta de dados em um único centro de Equoterapia. Assim não podendo generalizar nossos achados em todos os indivíduos com DI. 


\section{Conclusão}

O presente estudo demonstrou contribuição para o desenvolvimento e desempenho físico de crianças com DI. O esporte tem importância comprovada e relevante na melhora da qualidade de vida, favorece o desenvolvimento físico e é uma ferramenta poderosa para a inclusão social de pessoas com deficiência. Sugerimos para futuras pesquisas a avaliação com outros grupos de praticantes, como por exemplo pessoas com Síndrome de Down, Encefalopatia Crônica da Infância não Progressiva e idosos, assim como a reavaliação ao longo do tempo.

\subsection{Relevância Clínica}

Na prática clínica, testes para análise da flexibilidade muscular, da força muscular e do equilíbrio postural em individuos com DI podem nos permitir discriminar adequadamente aspectos importantes a serem considerados como melhorias para o tratamento com equoterapia no programa pré esportivo.

A identificação dessas propriedades permite que os profissionais envolvidos no contexto da equitação e da reabilitação por meio da equoterapia intervenham diretamente como um recurso valioso de suporte para a melhora desses parâmetros avaliados e inclusão de pessoas com DI em atividades esportivas. Os resultados obtidos a partir desse estudo também poderá ser utilizado como suporte e referência para futuras pesquisas.

\section{Agradecimentos}

Os autores agradecem pelo apoio financeiro a esta pesquisa fornecida pelo Conselho Nacional de Desenvolvimento Científico e Tecnológico para a Melhoria de Pessoal de Nível Superior (CNPq), a Fundação de Ensino e Pesquisa do Estado de Minas Gerais (FAPEMIG), a Fundação de Ensino e Pesquisa de Uberaba (FUNEPU) e Associação de Pais e Amigos dos excpecionais (APAE - Uberaba). Agradecemos também o incentivo e apoio incondional do Prof. Dr Vicente de Paula Antunes Teixeira, um grande pai científico. A colaboração de toda equipe de profissionais, equoterapeutas e de manejo equino do Centro de Equoterapia Dr Guerra da Apae de Uberaba que estiveram envolvidos com o projeto, em especial ao Cordenador Clínico e Diretor da instituição, Alex Abadio Ferreira. Aos praticantes que participaram do estudo e por último, mas não menos imporante, os nossos parceiros e essenciais colaboradores desse projeto, os cavalos.

\section{Referências}

Ande. (2021). O método equoterapia. Ande-Associação Nacional de Equoterapia.Brasil.http://equoterapia.org.br/articles/index/article_detail/142/2022.

Araújo, G. T. de, Lombardi, L. A., Santana, L. F., Silva, F. S., Dias, D. V \& Espindula, A. P. Base de dados atualizados de indicadores padronizados de citações de autores científicos na área de equoterapia: uma revisão bibliométrica. (2021). Research, Society and Development, 10 (4), e20110413191. 10.33448/rsd-v10i4.13191.

Bertolla, F., Baroni, B. M., Leal Junior, E. C. P \& Oltramari, J. D. (2007). Efeito de um programa de treinamento utilizando o método Pilates na flexibilidade de atletas juvenis de futsal. Revista Brasileira de Medicina do Esporte, (4), 222-226.

Carneiro, J. A. O., Grespan, T. E., Santos-Pontelli, T. E. G., Colafêmina, J. F., Colafêmina, J. F., Carneiro, A. A. O \& Ferriolli E. V. (2010). Análise do equilíbrio postural estático utilizando um sistema eletromagnético tridimensional. Braz J Otorhinolaryngol., 76 (6), $783-788$.

Eichinger, F. L. F., Soares, A.V., Carvalho Júnior, J. M., Gevaerd, M. S., Domenech, C \& Borges Júnior, N. G.(2016). Dinamometria lombar: um teste funcional para o tronco. Revista Brasileira de Medicina do Trabalho, São Paulo, v. 14 (2), 120-126.

Espindula, A. P., Assis, I. S. A. de., Simões, M., Ribeiro, M. F., Ferreira, A. A., Ferraz, P. F., Cunha, I. C., Ferraz, M. L. F., Sande De Souza, L. P., Bevilacqua Junior, D. E \& Teixeira, V. P. A. (2014). Material de montaria para equoterapia em indivíduos com síndrome de Down: estudo eletromiográfico. ConScientiae Saúde, 13 (3), 349-356.

Espindula, A. P., Fernandes, M., Ferreira, A. A., Ferraz, M. L. da F., Cavellani, C. L., Souza, L. A. P. S. de \& Teixeira, V. de P. A. (2012). Flexibilidade muscular em indivíduos com deficiência intelectual submetidos à equoterapia: estudo de casos. Rev. Ciênc. Ext. 8 (2), 125-133.

Espindula, A. P., Simões, M., Assis, I. S. A., Fernandes, M., Ferreira, A. A., Ferraz, P. F., Cunha, I. C., Ferraz, M. L. F., Sande De Souza, L. A. P \& Teixeira, V. P. A. (2012). Análise eletromiográfica durante sessões de equoterapia em praticantes com paralisia cerebral. ConScientiae Saúde, 11 (4), $668-676$. 
Research, Society and Development, v. 10, n. 7, e10410716198, 2021

(CC BY 4.0) | ISSN 2525-3409 | DOI: http://dx.doi.org/10.33448/rsd-v10i7.16198

Fernandes, M., Espindula, A. P., Bevilacgua Junior, D. E., Tolentino, J. A., Silva, F. R., Araújo, M. F., Ferreira, A. A \& teixeira, VPA. (2018). Activation of lower limb muscles with different types of mount in hippotherapy. Journal of Bodywork and Movement Therapies, 22, 52-56.

Gross, D. G. L., Nascimento, M. B. de M. T. do., Araújo, F. M. C., Gama, L., Saraiva, W. S \& Lisbôa Junior, I. M. (2019). Equoterapia para pessoas com deficiência: Uma conexão com a educação física. Revista Amazônia Science \& Health, 7 (4), 78-89.

Oliveira Júnior, E., Soeth, P. R., Paixão, A. F. V., Antunes, F, D \& Dias, F. (2018). Postural Balance in Children with Intellectual Disabilities. J Health Sci, $20(2), 140-5$.

Laje, J. B., Ribeiro, M. F., Teixeira, V. P. A., Rosa, R. C., Ferreira, A. A \& Espindula, A. P. (2020). Effect of horse riding equipment in activity of trunk and lower limb muscles in equine-assisted therapy. Acta scientiarum. Health sciences (ONLINE), 42, 1-8.

Moraes, A. G., Silva, M., Copetti, F., Abreu, A. C \& David, A. C. (2015). Equoterapia no controle postural e equilíbrio em indivíduos com paralisia cerebral: revisão sistemática. Rev Neurocienc, 23 (4), 546-554.

Pereira, E. L., Silva, C. F. da \& Mazo, J. Z. (2015). As primeiras participações de atletas do hipismo sul-rio-grandense em Jogos Olímpicos. Rev Bras Educ Fís Esporte, 29 (1), 47-64.

Pereira, A.S., Shitsuka, D.M., Parreira, F.J \& $\quad$ Shitsuka, R. (2018). Metodologia da pesquisa científica. UFSM.https://repositorio.ufsm.br/bitstream/handle/1/15824/Lic_Computacao_Metodologia-Pesquisa-Cientifica.pdf?sequence=1.

Ribeiro, M. F., Patrizzi, L. J., Teixeira, V. de P. A \& Espindula, A. P. (2016). Análise do equilíbrio e flexibilidade muscular de idosos submetidos a intervençãofisioterapêutica. Acta Scientiarum. Health Sciences, 38 (2), 129-136.

Schafer, A. A., Bitencourt, L. T. G., Cassetari, L. T. G., Uggioni, E. S \& Meller, F. O. (2019). Características pré e pós-natais de crianças e adolescentes com deficiência intelectual. Revista Cefac,. 21 (3), 1-9.

Schwartzman, J. S \& Lederman V. R. G. (2017). Deficiência intelectual: causas e importância do diagnóstico e intervenção precoces. Inclusão Social, 10 (2), $17-27$

Silva, D. A. da, Oliveira, K., Pereira, A. M \& Duarte, A. C. A. (2018). Cinética do passo e do trote de cavalos treinados com e sem rédea pessoa. Brazilian Journal of Biosystems Engineering, 12 (3), 228-233.

Silva, E. O., Azevedo, I. A \& Marques, M. C. S. (2019). A utilização do cavalo em paciente com transtorno do espectro autista: uma revisão integrativa. Brazilian Journal of Health Review, 2 (4), 3719-3728.

Suchomel T. J., Nimphius, S., Bellon C. R \& Stone M. H. (2018). The Importance of Muscular Strength: Training Considerations. Review Sports Med, 48(4), 765-785.

Tomaz, R. V. V., Santos, V. A., Avó, L. R. S., Germano, C. M. R \& Melo, D. G. (2017). Impacto da deficiência intelectual moderada na dinâmica e na qualidade de vida familiar: um estudo clínico-qualitativo. Cadernos de Saúde Pública, 33, (11), 1-14.

Wickert, H. O cavalo como instrumento cinesioterapéutico. Revista Equoterapia, (3): 03-14, 1999. 